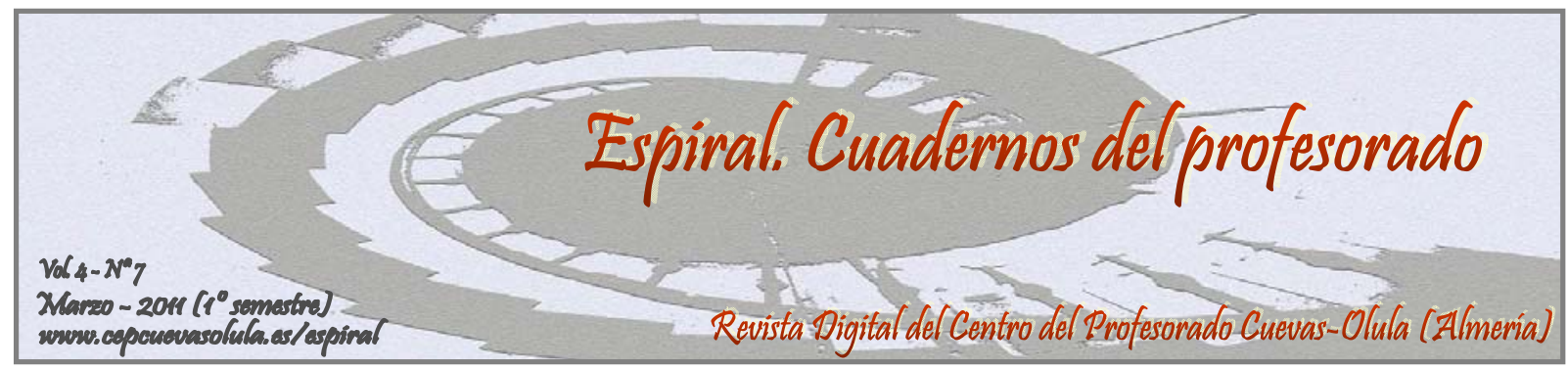

\title{
PROGRAMAS DIDÁCTICOS PARA EDUCACIÓN FÍSICA A TRAVÉS DE LA EDUCACIÓN DE AVENTURA
}

\section{EDUCATIONAL PROGRAMS FOR PHYSICAL EDUCATION THROUGH ADVENTURE EDUCATION}

\section{Antonio Baena Extremera}

\section{Facultad de Ciencias del Deporte, Universidad de Murcia, España}

RESUMEN: El presente trabajo, tiene como objetivos conocer las características de los diferentes programas de Educación y Aventura utilizados en educación física y diferenciar los pasos seguidos por cada uno de ellos. Se ha realizado una revisión documental explicativa, basándose en las principales bases de datos internacionales, obteniendo, un gran número de documentación. Tras seleccionar los artículos válidos y realizar los análisis documentales, se responde y discute a los objetivos de esta investigación.

Palabras clave: educación y aventura, aprendizaje experiencial, programas, educación física, deportes de aventura.

ABSTRACT: The present study aims to determine the characteristics of the different programs used in Adventure Education and Physical Education and differentiates the steps taken by each of them. It has been realized an explanatory documentary review, based on the main international databases, obtaining a large number of documents. After selecting the valid papers and tests documents, answers and discuss the aims of this research are answered and discussed.

Key words: adventure education, experiential learning, programs, physical education, adventure sports.

Baena-Extremera, A. (2011). Programas didácticos para Educación Física a través de la Educación de Aventura. Espiral. Cuadernos del Profesorado [en línea], 4(7), 3-13. Disponible en: http://www.cepcuevasolula.es/espiral. 


\section{1.- INTRODUCCIÓN A LA EDUCACIÓN DE VENTURA EN EDUCACIÓN FÍSICA.}

La Educación Física, al igual que el sistema educativo, está en continuo proceso de cambio, debido entre otros motivos, al cambio de la sociedad. A los alumnos, ya no les basta con aprender los contenidos tradicionales trabajados durante años centrados en la pista polideportiva y el pabellón, sino que buscan nuevos aprendizajes más acordes a sus intereses y necesidades. Entre estos intereses, se sitúan las actividades en la naturaleza y los deportes de aventura, contenidos que cada vez están adquiriendo más peso en las programaciones, desbancando a otros como los deportes colectivos, el atletismo, etc. (Granero, Baena y Martínez, 2010). Este proceso de cambio, ha llevado a que cada vez sean más los docentes de Educación Física e incluso de otras materias, los que incluyen en sus programaciones, juegos y actividades al aire libre, deportes en la naturaleza y deportes de aventura.

Cornell, Hadley, Sterling, Chan y Boechler (2001), especifican que el medio natural es un lugar lleno de estímulos para desarrollar a los alumnos, sobre todo a nivel psicológico. Las actividades realizadas por los niños y niñas durante sus infancias, (generalmente de tipo recreativas y de aventura), a menudo pueden ser entendidas como un tiempo de exploración, descubrimiento y de juego (Ewert, Place y Sibthorp, 2005), que les proporcionará interacción con un medio poco común lleno de estímulos que les harán crecer y madurar. Se tiene por tanto el consenso de muchos autores, de entender el aire libre y el medio natural, como el lugar favorito para las primeras actividades de la vida (Palmberg y Kuru, 2000), calificando ésta como, el primer aula escolar. Y es que siguiendo a Granero y Baena (2007), la naturaleza se puede considerar como la mayor aula de Educación Física del mundo, ya que presenta unas posibilidades educativas y de aprendizaje tan amplias, que desbancan a cualquier área curricular y escenario educativo.

El uso del medio natural como escenario facilitador de experiencias al aire libre con propósitos educativos, hasta llegar a la educación de aventura, presenta una larga tradición, sobre todo en Estados Unidos y que se puede remontar incluso hasta Platón (Neill, 2004; Outward Bound, 2006; Zmudy, Curtner-Smith y Steffen, 2009).
Se define la Educación de Aventura (EA) como el conjunto de actividades variadas que interaccionan con el entorno natural, conteniendo elementos de peligro real o aparente, donde el resultado es incierto, pudiendo ser influido por el participante y la circunstancia (Galloway, 2006; Gilbertson, Bates, McLaughlin, y Ewert 2006). En la educación al aire libre, se trabajan principalmente las relaciones inter e intrapersonales a través de tareas que implican a menudo, un reto con necesidad de resolver un problema (Priest y Gass, 2005). Conlleva una participación de los alumnos directa y activa, ofreciendo a los discentes experiencias de aprendizaje que les involucran en consecuencias reales (Prouty, Panucucci y Collinson, 2007). En resumen, podemos decir que la EA es un proceso mediante el cual los participantes intervienen en actividades, tareas y juegos con un componente de incertidumbre y cierto riesgo subjetivo, entremezclado con propósitos de confianza, reto, compañerismo, etc.

Otra forma de entender la EA, es a través de las situaciones experienciales. El aprendizaje de aventura o el Adventure Learning (Doering, 2006) es un enfoque donde se diseñan experiencias de aprendizaje que ofrecen a los estudiantes la oportunidad de explorar las cuestiones del mundo real a través del aprendizaje auténtico, donde será necesaria la colaboración. Para ello, se sitúa a los educandos en entornos poco familiares para ellos, para que vivan experiencias que les generen disonancias cognitivas y que den lugar a la resolución de problemas planteados a través de las actividades propias del área de Educación Física, consiguiendo además una reflexión personal y grupal. Apoyándose en la experiencia (Kolb, 1984) y el aprendizaje basado en la indagación (Bransford, Brown y Cocking, 1999; Dewey, 1938), los estudiantes se convierten en participantes activos, donde identifican y hacen preguntas, resuelven problemas del mundo real y toman medidas en su propio entorno. Como se aprecia, este tipo de prácticas, hace pensar que si los niños y niñas se enfrentan a situaciones límite para ellos en un entorno natural, serán capaces de crear un plan deliberado para solventar la situación (Rogoff, Gauvain y Gardner, 1987).

Así, en nuestro país, la EA y el aprendizaje de aventura, ha sido muy utilizado en otras versiones educativas, como el outdoor training (Jiménez y Gómez, 2008), los Juegos y Deportes de Aventura (Granero y Baena, 2011), los Juegos 
de Reto y Aventura (Gómez, 2008), o la Pedagogía de la Aventura (Parra, Caballero y Domínguez, 2009). Pero, ¿de donde proceden estos programas?

El origen de los programas de aventura se podría remontar a las prácticas del camping organizado y a los movimientos de la educación experiencial. Desde la creación de las Salem School en Alemania por Hahn hacia 1920, hasta su extensión con la Gordonstoun School en Escocia en 1934, pasando por el County Badge Scheme for Holt's Blue Funnel Shipping Company en 1940 y la primera Outward Bound School en Aberdovey (Gales) en 1941.

No obstante, la mayoría de los investigadores determinan el origen de la educación moderna de aventura a Kurt Hahn (1957), quien ideó hacia 1941, un programa para evitar las posibles pérdidas por muerte en una naviera, por hundimiento de sus buques. En los inicios de este programa, desarrollado durante un mes, se buscaba mejorar la independencia, la iniciativa, el desarrollo físico de los trabajadores,... e incluso el ingenio para sobrevivir. Tal fue el éxito de Hahn, que pronto se extendió sus enseñanzas por todo el mundo. Hoy en día, se puede apreciar cómo ha habido un aumento en el número de programas de EA durante los últimos 40 años. Se calcula que más de 200 programas de educación con salidas basada en la aventura se ofrecían en Estados Unidos sobre 1975 (Ewert, 1983). Una década más tarde, se hablaba de 542 programas relacionados con la naturaleza y la aventura ofrecidos por diferentes universidades de los EE.UU. (Hendee y Roggenbuck, 1984); y en 1994, llegaron a ser más de 40.000 estudiantes los que participaron en programas de este tipo, consolidándose en todo el mundo.

Hacia 1962, se crea la escuela Outward Bound en América del Norte, iniciando el movimiento de EA en los Estados Unidos. Esta escuela, fue seguida de cerca por la National Outdoor Leadership School (NOLS) en 1965, por el Project Adventure en 1971, y por la Wilderness Education Association sobre 1977. A mediados de la década de 1970, más de 190 programas de EA estaban operando ya en los Estados Unidos, realizándose más de la mitad de la programas en los centros universitarios. A partir de este momento, el número de programas de EA mostraron un crecimiento constante desde la década de 1970, 1980 y 1990, tras la creación de la Asociación para la Educación Experiencial
(AEE) en 1975 (Attarian, 2002). Hoy en día, esta asociación tiene representantes en más de 670 organizaciones.

En EE.UU., la inclusión de la EA y las actividades al aire libre como contenidos para el área de Educación Física se inició en la década de 1990. Esto llevó hasta la inclusión de la EA en la política educativa de otros países de manera oficial en sus documentos curriculares, como es el caso de Gran Bretaña (por ejemplo, las actividades al aire libre y de aventura se han incluido como parte del Plan de Estudios Nacional de Educación Física); o en Australia, donde la cantidad del tiempo asignado para las actividades de aventura en los programas escolares de Educación Física ha aumentado en los últimos años con el objetivo de promover la recreación y la participación en actividad física (Lugg y Martin, 2001; Pickett y Polley, 2001).

La consistencia que han demostrado estos programas a nivel educativo ha sido tal, que a lo largo de la historia se han realizado numerosas investigaciones entorno a la EA. Por ejemplo, Marsh y Richards (1988), realizaron un trabajo con 66 estudiantes de noveno grado con bajos niveles académicos, aplicándoles un programa de 6 semanas de aventura, con el objetivo de mejorar el rendimiento académico. Al final del mismo, se encontraron efectos positivos en el logro académico y el autoconcepto de los logros. Otros investigadores también han demostrado que los programas de aventura puede ser útil para motivar a los estudiantes para mejorar su rendimiento académico (Gass, 1987; Gillenson, 1983; Hammerman, 1978; Stogner, 1978).

Sakofs y Schuurman (1991), realizaron una investigación con jóvenes de 13 a 18 años, con un programa diseñado en el desierto, con búsqueda de efectos terapéuticos. El grupo experimental tuvo resultados significativamente más positivos que el grupo control en 10 de las 33 escalas psicométricas y evaluaciones de comportamiento realizadas: locus de control, orientación asociales, agresividad manifiesta, orientación de los valores, la inmadurez, la depresión de abstinencia, la ansiedad social, la represión, etc.

Otro ejemplo es el realizado por Gatzemann, Schweizer y Hummel (2008), quienes en un programa de 8 días de EA (lo que podría ser una unidad didáctica en un centro educativo), obtuvieron resultados positivos, al trabajar contenidos de actividades de aventura y aire 
libre, como senderismo, orientación, ciclismo de montaña, kayak, culminando con un viaje de varios días. Según estos autores, estos contenidos concuerdan perfectamente con la organización curricular de los colegios, seguida por los profesores de Educación Física. aquellos artículos científicos aceptados y/o publicados por las revistas internacionales más importantes del mundo, cuyo rigor científico es ampliamente conocido.

\section{3.- Procedimiento.}

Tabla 1: Número de artículos encontrados.

\begin{tabular}{lcccc}
\hline & $\begin{array}{c}\text { Adventure } \\
\text { education }\end{array}$ & $\begin{array}{c}\text { Adventure } \\
\text { learning }\end{array}$ & Adventure sport & Válidos \\
\hline Isi Web of Knowledge & 62 & 10 & 2 & $12,8,0$ \\
Scopus & 50 & 46 & 19 & $35,16,2$ \\
SportsDiscus & 155 & 23 & 113 & $41,11,15$ \\
Total año 2010 & 9 & 9 & 1 & \\
Total año 2009 & 2 & 5 & 3 & \\
Total año 2008 & 2 & 6 & 1 & \\
Total año 2007 & 1 & 3 & 2 & \\
Total año 2006 & 3 & 3 & 3 & \\
\hline
\end{tabular}

Una vez revisada la literatura existente, el planteamiento de los objetivos de esta investigación se centran en conocer las características de los diferentes programas de EA utilizados en educación. Posteriormente, se pretende analizar algunos de los enfoques que ha recibido la EA en los diferentes modelos de enseñanza utilizados en los diferentes países.

\section{2.- METODO.}

\section{1.- Diseño.}

El diseño llevado a cabo para esta investigación, ha sido la revisión documental descriptiva (Sampieri, Collado y Lucio, 1998), de la bibliografía científica más importante a nivel internacional relacionado con la temática, para desarrollar un análisis descriptivo en función de los objetivos establecidos.

\section{2.- Instrumentos y fiabilidad documental.}

Para llevar a cabo la revisión documental, se han utilizado algunos de los buscadores más importantes en investigación, como Isi Web of Knowledge, Scopus y SportsDiscus. Para ello, los descriptores introducidos para realizar dicha búsqueda, han sido:

- Adventure education.

- Adventure learning.

- Adventure sport.

En relación a la fiabilidad documental, hay que destacar que al realizar la revisión documental a partir de los resultados obtenidos en estas bases de datos, estamos asegurando una alta fiabilidad científica. Esto es debido a que en estas bases de datos, se recogen exclusivamente
Es necesario apuntar que, dependiendo del lugar desde donde se haga la búsqueda y del tipo de inscripción de revistas que se disponga, puede modificar el número de registros encontrados. Además, una vez realizada la búsqueda, se ha seleccionado los artículos según los siguientes indicadores:

- Por fechas: se han seleccionado los más recientes, teniendo en cuenta exclusivamente aquellos artículos antiguos de interés particular.

- Tipo de documento: se han seleccionado preferentemente, artículos de revista de carácter educativo.

- Idioma: la primera selección, ha sido utilizar artículos en inglés, posteriormente español, y luego otros idiomas como francés y alemán.

Una vez seleccionado los artículos a revisar, se ha procedido a llevar a cabo un análisis de cada uno, para poder responder a los objetivos de la investigación.

\section{3.- RESULTADOS.}

\section{1.- Análisis bibliométricos.}

Una vez realizadas las búsquedas bibliográficas, los resultados encontrados en relación a los artículos, quedan reflejados en la tabla 1.

\section{2.- Primer objetivo.}

Tras revisar la documentación encontrada para responder a nuestro objetivo y siguiendo a Hattie, Marsh, Neill y Richards (1997), podemos establecer que las características comunes de los programas de aventura son: 
1. Realización de actividades fuera de lo común, en ambientes desconocidos. Numerosos investigadores han determinado la necesidad de trabajar en espacios desconocidos para los participantes en los programas de EA, (Kimball y Bacon, 1993; Nadler, 1993; Walsh y Golins, 1976), y donde las actividades propuestas sean totalmente nuevas para el alumnado.

2. Trabajar con grupos reducidos, por lo general, menos de 16 personas. Según Walsh y Golins (1976), el tamaño del grupo se cree que es una característica importante en la determinación de la eficacia de un programa de EA. Es lo suficientemente grande como para que exista diversidad y posibles conflictos y lo suficientemente pequeño para evitar las agrupaciones entre alumnos.

3. Trabajar actividades que planteen objetivos mentales y físicos. Según Schoel, Prouty y Radcliffe (1988), se suelen trabajar actividades que trabajan la confianza y la empatía, la comunicación con los demás, la toma de decisiones para solventar problemas, la responsabilidad social y por supuesto, actividades de responsabilidad personal. Conforme el grupo comienza a trabajar, los alumnos descubren las necesidades y peculiaridades de cada uno de los miembros, con lo que a consecuencia de ello, aprenden a trabajar en equipo para reforzar los puntos débiles, y aprovechar los puntos fuertes grupo (Hopkins y Putnam, 1993). De esta forma, se equilibran las necesidades individuales con las necesidades grupales. Tan importante es la formación y cohesión del grupo, que según las investigaciones de Witman (1995), muchos alumnos describen como uno de los aspectos más importantes en estos programas, el "sentirse parte del grupo".

4. Producir interacciones frecuentes $\mathrm{e}$ intensas a través de pruebas que impliquen la resolución de problemas en grupo tomando decisiones.

5. Plantear una duración de 2 a 4 semanas como mínimo.

Una de las características que quiero destacar, son referentes a las actividades y tareas, ya que son uno de los aspectos más importantes en estos programas. Las diversas investigaciones (Gass, 1995; Luckner y Nadler, 1997) indican que una serie de actividades de desafío pueden conducir a conseguir resultados positivos que se asocian típicamente con la EA. Por ejemplo, actividades como puentes colgantes, escalada o piragüismo, son utilizadas como herramientas para promover el crecimiento de los participantes dentro de estos programas (Marsh, Richards y Barnes, 1986). Con estas actividades, se busca situar a los discentes en una situación de disonancia que si la superan, llevarían al participante a mejorar su crecimiento personal (Conrad y Hedin, 1981; Dyson, 1995; Iso-Ahola y Graefe, 1988; Witman, 1995). Para superar dichas actividades, los alumnos deberán poner en juego todas sus capacidades mentales y físicas, de manera que cada vez la dificultad de las tareas y el esfuerzo de los alumnos vayan aumentando.

Según Bisson (1998), la secuencia de las actividades incluidas en un programa está estrechamente relacionada con la eficacia del programa. Walsh y Golins (1976) sostienen que las actividades incluidas en un programa de EA deben estar bien organizadas con el fin de provocar en los participantes los efectos educativos buscados. Es indispensable conocer bien a los alumnos y partir del nivel inicial de ellos, pues si comenzamos con tareas demasiado fáciles se aburrirán, y si son demasiado difíciles, el programa no tendrá su efecto. Para ello, se deben diseñar las actividades de forma que todos los discentes alcancen el éxito, pues de esta manera, se aumenta los efectos positivos del programa (Kiewa, 1994; Nadler, 1993; Walsh y Golins, 1976). Ejemplo claro lo podemos encontrar en el trabajo de Iso-Ahola y Graefe (1988), quienes demostraron que los deportistas que obtenían éxito en la escalada en roca, aumentaban de manera significativa su autoestima. Sobre esto, hay que decir que según Kimball y Bacon (1993), en los programas de EA, las actividades suelen estructurarse de manera que el éxito y el dominio son posibles, pero sobre todo probables. Aunque, hay que comentar que el fracaso también tiene sus efectos positivos en los programas de aventura.

\section{3.- Segundo objetivo.}

En relación al segundo objetivo de esta investigación y siguiendo a Bacon (1987), se pueden describir tres posibles modelos de EA:

1. En el Mountains Speak for Themselves. En este modelo, los participantes son responsables de reflexionar por su propia cuenta sobre la experiencia de EA. Durante este modelo, los instructores no realizan ningún debate y/o retroalimentación.

2. En el Outward Bound Plus, el profesor tiene las funciones del líder de la discusión, es 
consejero y facilitador del proceso grupal. Este modelo se centra en crear vínculos entre las actividades y la vida de los participantes cada día, y estimula la reflexión y el debate.

3. El tercer modelo, es el modelo metafórico, en que las actividades son conscientemente formuladas de manera que se convierten en metáforas de la experiencia para los desafíos en la vida de los participantes durante cada día. Este modelo se basa en las características de los otros dos modelos con la inclusión de la experiencia directa, la reflexión y la discusión. El modelo metafórico es considerado por algunos como el más eficaz (Bacon, 1987; Gass 1993).

Además, tras realizar el análisis documental, se ha podido comprobar la existencia de aplicaciones variadas de modelos de programas de aventura en poblaciones diferentes. Por lo que, teniendo en cuenta los modelos anteriores, se han ido desarrollando multitud de programas de EA. Por ejemplo, entre los programas más importantes, destacamos el programa de aprendizaje experiencial de Kolb (1984, en Evans, Forney y Guido-DiBrito, 1998), o el de la de Outward Bound. A parte de éstos, hay que destacar que uno de los más desarrollados es el establecido por Rhonke (1989), en el que las actividades al aire libre y de aventura (por ejemplo, kayak, piragüismo, senderismo, buceo) se utilizan como un medio a través del cual, el alumno debe conseguir metas físicas y psicosociales.

Cada programa, establece unas etapas de aprendizaje diferentes. Por ejemplo, en el programa de Rhonke (1989), los estudiantes están obligados a moverse a través de siete etapas:

1. Actividades con desplazamientos desconocidos.

2. Actividades para romper sus inhibiciones.

3. Actividades de comunicación.

4. Actividades para resolver problemas.

5. Tareas para crear confianza y que puedan afrontar los retos del grupo.

6. Actividades con elementos y situaciones con cuerdas.

7. Actividades con problemas individuales, donde se incluyen elementos con cuerdas a cierta altura.

Según Gómez (2008), en relación a las actividades de reto y aventura, las fases que se proponen para llevar a cabo un programa de este tipo, serían las siguientes:

1. Fase Experimental, donde el alumnado se enfrenta, solo y en compañía, a una sucesión de situaciones que le exigirán enfrentarse a diferentes tareas que le obligarán a tener que comunicarse con sus compañeros y encontrar soluciones. Las tareas de estas fases, se organizan en tres niveles:

a) Juegos cooperativos: donde el objetivo es conocer al grupo y divertirse.

b) Juegos de confianza no tradicionales: donde el objetivo es el desarrollo de la confianza y la cohesión de grupo.

c) Actividades de compromiso de grupo: donde se busca la solución de los problemas planteados y la propuesta y localización de las soluciones correctas por parte del grupo.

2. Fase de Reflexión, que estaría constituida por dos puntos clave, que son la reflexión y la transferencia; aquí el profesor se convierte, según Gómez (2008, p.8) en un facilitador que idealmente debe poseer conocimientos y recursos sobre dinámica de grupos, habilidades de coaching, toma de decisiones, etc. en relación al mundo de los jóvenes y su problemática.

Siguiendo a Parra, Caballero y Domínguez (2009), y desde una perspectiva más experiencial, establecen como fases en su programa de aventura los siguientes pasos metodológicos:

1. Actividades de presentación, distensión, familiarización y conocimiento.

2. Actividades de redescubrimiento sensorial y habilidades básicas.

3. Actividades de afirmación, autovaloración individual o colectiva.

4. Actividades de comunicación y cooperación simple.

5. Actividades de confianza y técnicas básicas.

6. Actividades de cooperación compleja y resolución de problemas.

7. Actividades de instinto, iniciativa y decisión.

8. Actividades de aventura compleja. Actividades deportivas.

9. Actividades de asimilación y reflexión. 
Desde mi punto de vista, las fases para trabajar con un programa de EA, así como las fases para el aprendizaje experiencial de actividades y deportes de aventura, va a depender de ciertas variables, como por ejemplo, el tipo de alumnado, conocimientos y experiencias previas, tipo de material e instalaciones disponibles, objetivos finales del programa, etc. De manera general, las fases para un grupo de alumnos con ninguna o baja experiencia-conocimiento en deportes y actividades de aventura, serían:

$1^{a}$ Fase: Fase Experiencial y de Conocimiento:

1.1. Actividades de conocimiento de sí mismo y sus posibilidades.

1.2. Actividades de conocimiento de los demás y de las posibilidades como grupo de trabajo.

1.3. Actividades de conocimiento del material e instalaciones específicos. Confianza en él.

1.4. Actividades de reflexión grupal y autorreflexión.

Dentro de esta fase, el orden ideal de planteamiento de las actividades iría orientado tal y como he especificado, pero con la salvedad, de que lo ideal sería intercalar actividades de los cuatro tipos, no hacerlas por separado. Para ello, es muy interesante hacer uso de una metodología muy participativa por parte del alumnado, basado en estilos de enseñanza cognoscitivos, como el aprendizaje por problemas (ABP) o ProblemBased Learning, ya que potenciaran el aprendizaje significativo de los mismos. " $E l$ ABP es un método mediante el cual los alumnos construyen su conocimiento sobre la base de los problemas de la vida real. Es decir, no se trata de resolver problemas y encontrar la solución acertada sobre la información proporcionada previamente, sino todo lo contrario" (Font, 2004, p.84). Para ello, las sesiones deben estar organizadas para trabajar en pequeños grupos de alumnos, los cuáles deberán analizar y resolver los problemas seleccionados o diseñados especialmente para el logro de los objetivos de aprendizaje. Trabajando de esta forma, se busca en los alumnos además del aprendizaje del conocimiento propio, que puedan elaborar un diagnóstico de sus propias necesidades de aprendizaje, y que comprendieran la importancia de trabajar individual o colaborativamente, desarrollando habilidades de análisis y síntesis de información (fundamental en la gestión del riesgo en estos deportes), además de comprometerse con su proceso de aprendizaje.

$2^{a}$ Fase de Práctica. En el caso de alumnado que tengan experiencias y conocimientos en estas actividades y deportes, se podría empezar directamente por esta fase, o bien trabajar algunos de los apartados anteriores para recordar y afianzar, según la evaluación inicial realizada. Pasos:

2.1. Actividades de reto y problemas con modificación de la situación real, donde se utilicen los conocimientos adquiridos en las fases anteriores. Se recomienda avanzar en los aspectos teóricos y técnicos de materiales, instrumentos, utilidades, etc.

2.2. Actividades de reto y problemas con pequeñas modificaciones de la situación real.

2.3. Práctica analítica de la situación real.

2.4. Actividades de reto y problemas con situación global real.

2.5. Actividades de reflexión grupal y autorreflexión.

En esta fase, al contrario que la anterior, no es tan recomendado hacer una sesión donde se vayan mezclando los cinco pasos, sino que se podría ir desde el primero al último sin mezclar sus tipos de actividades.

\section{4.- DISCUSIÓN Y CONCLUSIONES.}

Las actividades en la naturaleza, los deportes de aventura y sus aplicaciones didácticas y educativas, han sido bien estudiados por diversos autores (Anglada, 2008a, 2008b; Bronson, Gibson, Kishar y Priest, 1992; Granero, Baena y Martínez, 2010, Granero y Baena, 2011, Hatch y McCarth, 2005; entre otros), dando lugar a diversos programas y propuestas a lo largo del mundo. Entre ellos, la EA se ha convertido hoy en día, en un sistema ampliamente reconocido y aceptado como campo profesional que promueve muchas aspectos positivos de los educandos (Peter, 2004; Wurdinger y Steffen, 2003).

Fabrizio y Neill (2005) sostienen que el comienzo de una experiencia de aventura al aire libre se caracteriza por los sentimientos de excitación, anticipación y euforia en la mayoría de los participantes (Sutherland y Stroot, 2010). El denominador común más notable de los programas de aventura es la implicación de tareas físicamente activas y fuera del ambiente normal de la persona, de su trabajo o de su vida. Como 
se ha explicado, las actividades son muy variadas e incluyen tareas de exploración, cursos de montaña, ejercicios de supervivencia, cursos de desierto, entre otros (ver Hogan, 1968).

Los principales objetivos de los programas EA, se centran en fomentar el desarrollo emocional, físico y social, a través del aumento de la autoestima, la conciencia de sí mismo, la confianza en uno mismo, la mejora de las habilidades de comunicación, la cooperación con los demás, y sobre todo, las habilidades para resolver problemas (Bisson, 1998; Prouty, 1999). Por ejemplo, algunos programas de aventura han sido diseñados específicamente para producir mejoras a nivel cognitivo, especialmente en el lenguaje y las matemáticas, a través de un programa integrado de apoyo escolar. Es importante señalar que ciertos programas de aventura tienen objetivos específicos en relación con las metas académicas, mientras que la mayoría no lo hacen.

Los programas de EA, son además utilizados con efectos muy beneficiosos en poblaciones muy diversas. Por ejemplo, la mayoría de los participantes en los programas de aventura de la escuela Outward Bound, han sido estudiantes o gerentes, aunque hay que destacar que algunos programas, contemplan específicamente a los adolescentes con comportamiento alterado, pacientes psiquiátricos, alcohólicos en recuperación, pacientes con esquizofrenia, personas con trastorno del estado de ánimo, delincuentes, personas con problemas de alcohol y adolescentes con abuso de drogas (Gass y McPhee, 1990; Golins, 1979; Kelly y Baer, 1968; Sachs y Miller, 1992; Stich, 1983; Wright, 1982).

Se ha investigado, cómo los niños de diferentes edades desarrollan estrategias cognitivas interactuando con el medio natural, para por ejemplo, saber volver a casa después de una ruta de senderismo. Además, se descubrió que a partir de los ocho años, los niños comienzan a utilizar la estructuración espacial como sistema de navegación en los entornos naturales (Cornell et al., 2001). Si observamos los contenidos descritos para Educación Física por el Real Decreto 1513/2006 de enseñanzas mínimas, podemos ver cómo coincide el tratamiento de la estructuración espacial con estas edades. Por lo que sería ideal, trabajar con actividades y prácticas de senderismo para desarrollar este contenido en nuestros alumnos.
Conley, Caldarella y Young (2007) indican que tras un programa de EA con actividades de riesgo para alumnos de educación secundaria internos en situación de riesgo, se produjeron mejoras en la participación y más de la mitad de los estudiantes llegaron a aprender los contenidos del aula que antes creían que serían incapaces. Igualmente, Gehris, Kress y Swalm (2010), obtuvieron que la mayoría de su alumnado afirmara mejorar su aptitud física tras un programa de aventura, mejorando la fuerza y la condición física en general, aunque consideran que la resistencia podría disminuir. Pero estos mismos autores, llegaron a la conclusión de que muchos estudiantes decidieron practicar actividades de aventura como la escalada, fuera del colegio, una vez que se les había iniciado gracias a los programas de aventura. Esto nos hace pensar que, los alumnos practican en su tiempo libre las actividades y deportes que han aprendido en su centro educativo. Si como se ha dicho al inicio del artículo, las sociedades están cambiando, es hora de que las programaciones y contenidos trabajados por los profesores, comiencen a cambiar.

Por último, en la aplicación de los programas de EA, es necesario buscar un equilibrio entre la técnica y la experiencia dentro de las actividades. Independientemente del modelo y/o pasos metodológicos que sigamos, es crucial introducir aspectos cognitivos dentro de las actividades (usos de los materiales, técnicas de uso correctas,...) para intentar transferir el aprendizaje de los alumnos a sus futuras prácticas de ocio. Si nos dedicamos a dar exclusivamente sensaciones y experiencias, pero sin crecimiento técnico en estos deportes, estaremos desaprovechando la oportunidad de que los alumnos comiencen a practicar por su cuenta y de una forma segura.

\section{5.- REFERENCIAS BIBLIOGRÁFICAS.}

Anglada, P. (2008a). Investigaciones realizadas en torno a los programas de aventura. En L. Ariza Vargas y M. Guillén del Castillo (Coord), Actas del IV Congreso Internacional y XXV Nacional de Educación Física, [CD-ROM]. Córdoba: Universidad de Córdoba. [21-11-09].

Anglada, P. (2008b). Origen de los programas de aventura en la naturaleza con fines educativos. En L. Ariza Vargas y M. Guillén del Castillo (Coord), Actas del IV Congreso Internacional y XXV Nacional de Educación Física, [CD-ROM]. Córdoba: Universidad de Córdoba. [21-11-09]. 
Attarian, A. (2002). Trends in Outdoor Adventure Education. Preserving the past, pretecting the future. 16TH Annual International Conference on Outdoor Recreation and Education. Charleston County Park and Recreation Commission Charleston, SC October 24-31.

Bacon, S. (1987). The career beginnings Outward Bound component: An empirical evaluation. Greenwich, CT: Outward Bound USA.

Bisson, C. (1998). The effects of sequencing adventure activities on the development of group cohesion. Doctoral Dissertation presented at the 26th Annual Association for Experiential Education International Conference. Lake Tahoe: NV.

Bransford, J., Brown, A., \& Cocking, R. (1999). How people learn: Brain, mind, experience, and school. Washington, DC: National Academy Press.

Bronson, J., Gibson, S., Kishar, R. \& Priest, S. (1992). Evaluation of team development in a corporate adventure training program. Journal of Experiential Education, 15(2), 50-53.

Conrad, D. \& Hedin, D. (1981). National assessment of experiential education: Summary and implications. Journal of Experiential Education, 4(2), 6-20.

Conley, L., Caldarella, P. \& Young, E. (2007). Evaluation of a ropes course experience for atrisk secondary school students. Journal of Experiential Education, 30(1), 21-35.

Cornell, E.H., Hadley, D.C., Sterling, T.M., Chan, M.A. \& Boechler, P. (2001). Adventure as a stimulus for cognitive development. Journal of Environmental Psychology, 21, 219-231.

Dewey, J. (1938). The school and society. In M. Dworkin (Ed.), Dewey on education New York: Teachers College Press.

Dyson, B. (1995). Students' voices in two alternative elementary physical education programs. Journal of Teaching in Physical Education, 14, 394-407.

Doering, A. (2006). Adventure Learning: Transformative hybrid online education. Distance Education, 27(2), 197-215.

Evans, J.N., Forney, D.S. \& Guido-DiBrito, F. (1998). Student development in college: theory, research, and practice. San Francisco, CA: Jossey Bass.

Ewert, A. (1983). Outdoor adventure and selfconcept: A research analysis. Eugene: University of Oregon, Center of Leisure Studies.

Ewert, A., Place, G. \& Sibthorp, J. (2005). Early-Life Outdoor Experiences and an Individual's Environmental Attitudes. Leisure Sciences, 27, 225-239.

Fabrizio, S.M., \& Neill, J.T. (2005). Cultural adaptation in outdoor programming. Australian Journal of Outdoor Education, 9(2), 44-56.
Font, A. (2004). Líneas maestras del Aprendizaje por Problemas. Revista Interuniversitaria de Formación del Profesorado, 18(1), 79-95.

Galloway, S. (2006). Adventure recreation reconceived: Positive forms of deviant leisure. Leisure/Loisir, 30, 219-232.

Gass, M.A. (1993) Adventure therapy: therapeutic applications of adventure programmingq. Dubuque, IA: Kendall/Hunt.

Gass, M.A. (1987). The effects of a wilderness orientation program on college students. Journal of Experiential Education, 10(2), 30-33.

Gass, M.A. (1995). Adventure family therapy: An innovative approach answering the question of lasting change with adjudicated youth? Monograph on Youth in the 1990s, 4, 103-117.

Gass, M.A. \& McPhee, P.J. (1990). Emerging for recovery: Descriptive analysis of adventure therapy for substance abusers. Journal of Experiential Education, 13, 29-35.

Gatzemann,T., Schweizer, K. \& Hummel, A. (2008). Effectiveness of sports activities with an orientation on experiential education, adventurebased learning and outdoor-education. Kinesiology, 40(2), 146-152.

Gehris, J., Kress, J. \& Swalm, R. (2010). Students' Views on Physical Development and Physical Self-Concept in Adventure-Physical Education. Journal of Teaching in Physical Education, 29, 146-166.

Gilbertson, K., Bates, T., McLaughlin, T. \& Ewert, A. (2006). Outdoor education: methods and strategies. Champaign, IL: Human Kinetics.

Gillenson, A.J. (1983). Short term experiential laboratories in academic courses. Hanover, $\mathrm{NH}$ : Unpublished technical report, Dartmouth College.

Golins, G. (1979). Utilizing adventure education to rehabilitate juvenile delinquents. Denver, CO: Colorado Outward Bound.

Gómez, V. (2008). Juegos y actividades de reto y aventura en el contexto escolar (en línea). Wanceulen: Educación Física Digital, 4, 1-12.

Granero, A. \& Baena, A. (2007). Importancia de los valores educativos de las actividades físicas en la naturaleza. Habilidad Motriz: revista de ciencias de la actividad física y del deporte, 29, 5-14.

Granero, A. \& Baena, A. (2011). Juegos y deportes de aventura en la formación permanente del profesorado. International Journal of Medicine and Science of Physical Activity and Sport, (in press).

Granero, A., Baena, A. \& Martínez, M. (2010). Contenidos desarrollados mediante las activididades en el medio natural de las clases de Educación Física en secundaria obligatoria. 
Revista Ágora para la Educación Física y el Deporte, 12(3), 273-288.

Hammerman, D. (1978). Outdoor education. Journal of Physical Education and Recreation, 7, 28.

Hatch, K. \& McCarth, C. (2005). Exploration of challenge course long-term effects on members of collage student organizations. Journal of Experiential Education, 27(3), 245-264.

Hattie, J., Mars, H.W., Neill, J.T. \& Richards, G.E. (1997). Adventure Education and Outward Bound: Out-of-Class Experiences That Make a Lasting Difference. Review of Educational Research Spring, 67(1), 43-48.

Hendee, J. \& Roggenbuck, J. (1984, August). Wilderness-related education as a factor increasing demand for wilderness. Paper presented at the International Forest Congress Convention, Quebec City, Canada.

Hogan, J.M. (1968). Impelled into experiences: The story of the Outward Bound schools. London: Educational Productions.

Hopkins, D. \& Putnam, R. (1993). Personal growth through adventure. London, England: David Fulton Publishers.

Iso-Ahola, S.E. \& Graefe, A.R. (1988). Perceived competence as a mediator of the relationship between high risk sports participation and selfesteem. Journal of Leisure Research, 21(1), 3239.

Jiménez, P.J. \& Gómez, V. (2008). Turismo Activo y Outdoor Training: Metodología. Adventure Sport Tourism and Outdoor Training: Methodology. International Journal of Sport Science, 13, 69-79.

Kelly, F. J., \& Baer, D. J. (1968). Outward Bound schools as an alternative to institutionalization for adolescent delinquent boys. Boston, MA: Outward Bound, Inc.

Kiewa, J. (1994). Self-control: The key to adventure? Towards a model of the adventure experience. In E. Cole, E. Erdman, \& E. D. Rothblum (Eds.), Wilderness therapy for women: The power of adventure (pp. 29-41). Binghamton, NY: The Haworth Press, Inc.

Kimball, R.O. \& Bacon, S.B. (1993). The wilderness challenge model. In M. A. Gass (Ed.), Adventure therapy: Therapeutic applications of adventure programming (pp. 11-41). Dubuque, IA: Kendall/Hunt Publishing Company.

Kolb, D. (1984). Experiential learning: Experiences as the source of learning and development. Englewood Cliffs, NJ: Prentice Hall.

Luckner, J.L. \& Nadler, R.S. (1997). Processing the experience: Strategies to enhance and generalize learning. Dubuque, IA: Kendall/Hunt Publishing Company.
Lugg, A. \& Martin, P. (2001). The nature and scope of outdoor education in Victorian schools. Australian Journal of Outdoor Education, 5(2), 42-48.

Marsh, H.W. \& Richards, G.E. (1988). The Outward Bound bridging course for low-achieving high school males: Effect on academic achievement and multidimensional self-concepts. Australian Journal of Psychology, 40, 281-298.

Marsh, H.W., Richards, G.E. \& Barnes, J. (1986). Multidimensional self-concepts: The effect of participation in an Outward Bound program. Journal of Personality and Social Psychology, 50(1), 195-204.

Nadler, R.S. (1993). Therapeutic process of change. In M. A. Gass (Ed.) Adventure therapy: Therapeutic applications of adventure programming (pp. 5769). Dubuque, IA: Kendall/Hunt Publishing Company.

Neill, J.T. (2004) Experiential learning cycles. [en línea]. Disponible en: http://www.wilderdom.com/theory/ExperientialL earningCycles.html. [Consulta: 25 de marzo de 2011].

Outward Bound (2006). The history of outward bound. [en línea]. Disponible en: http://www.outwardbound.org/lic_sub3_history.h tm. [Consulta: 25 de marzo de 2011].

Palmberg, I.E. \& Kuru, J. (2000). Outdoor activities as a basis for environmental responsibility. Journal of Environmental Education, 31(4), 32-36.

Parra, M., Caballero, P. \& Dominguez, G. (2009). Pedagogía de la Aventura. In $\mathrm{M}^{\mathrm{a}}$ E., García Montes (coord.), Dinámicas y estrategias de recreación, (pp. 199-260), Barcelona: Graó.

Peter, M. (2004) Outdoor adventure in promoting relationships with nature. Australian Journal of Outdoor Education, 8(1), 20-28.

Picket, B. \& Polley, S. (2001) Investigating the history of outdoor education in South Australia. Australian Journal of Outdoor Education, 5(2), 49_53.

Priest, S. \& Gass, M. (2005). Effective leadership in adventure programming. Champaign, IL: Human Kinetics.

Prouty, D. (1999). Project adventure: A brief history. In J.C. Miles \& S. Priest (Eds.), Adventure programming (pp. 93-101). State College, PA: Venture Publishing.

Prouty, D., Panicucci, J. \& Collinson, R. (Eds.) (2007) Adventure education: theory and applications. Champaign, IL: Human Kinetics

Real Decreto 1513/2006, de 7 de diciembre, por el que se establecen las enseñanzas mínimas de la Educación Primaria. 
Rogoff, B., Gauvain, M. \& Gardner,W. (1987). The development of children's skills in adjusting plans to circumstances. In S. Friedman, E. Scholnick \& R. Cocking (Eds), Blueprints for Thinking: The Role of Planning in Cognitive Development, (pp. 303-320). New York, NY: Cambridge University Press.

Rhonke, K. (1989). Cowstails and Cobras II. Dubuque, IA: Kendall/Hunt.

Sakofs, M. \& Donna, S. (1991). Assessing the Impact of Wilderness Alternative for Youth Program: An Outward Bound Program for Adjudicated Youth. Outward Bound: Inc.

Sampieri, R.H., Collado, C.F. \& Lucio, P.B. (1998) Metodología de la investigación. Segunda edición. México: Mcgraw-Hill Interamericana Editores.

Sachs, J.J. \& Miller, S.R. (1992). The impact of a wilderness experience on the social interactions and social expectations of behaviorally disordered adolescents. Behavioral Disorders, 17, 89-98.

Schoel, J., Prouty, D. \& Radcliffe, P. (1988). Islands of healing: A guide to adventure based counseling. Hamilton, MA: Project Adventure, Inc.
Stogner, J. (1978). The effects of a wilderness experience on self-concept and academic performance (Doctoral dissertation, Virginia Polytechnic Institute and State University). Dissertation Abstracts International, 39, 4704A.

Stich, T.F. (1983). Experiential therapy for psychiatric patients. Journal of Experiential Education, 5(3), 23-30.

Walsh, V. \& Golins, G. (1976). The exploration of the Outward Bound process. Denver, CO: Colorado Outward Bound School.

Witman, J.P. (1995). Characteristics of adventure programs valued by adolescents in treatment. Monograph on Youth in the 1990s, 4, 127-135.

Wright, A. (1982). Therapeutic potential of the Outward Bound process: An evaluation of a treatment program for juvenile delinquents (Doctoral dissertation, Pennsylvania State University). Dissertation Abstracts International, 43, 923A.

Wurdinger, S. \& Steffen, J. (2003). Developing challenge course programs for schools. Dubuque, IA: Kendall Hunt.

Zmudy, M.H., Curtner-Smith, M.D. \& Steffen, J. (2009). Student participation styles in adventure education. Sport, Education and Society,14(4), 465-480. 